\title{
Keap1/Nrf2 pathway in kidney cancer: frequent methylation of KEAP1 gene promoter in clear renal cell carcinoma
}

\author{
Federico Pio Fabrizio ${ }^{1, *}$, Manuela Costantini' ${ }^{2,5,4, *}$, Massimiliano Copetti ${ }^{3}$, Annamaria \\ la Torre $^{1}$, Angelo Sparaneo ${ }^{1}$, Andrea Fontana ${ }^{3}$, Luana Poeta ${ }^{4}$, Michele Gallucci ${ }^{5}$, \\ Steno Sentinelli6, Paolo Graziano7, Paola Parente ${ }^{7}$, Vincenzo Pompeo5 , Laura De \\ Salvo6, Giuseppe Simone ${ }^{5}$, Rocco Papalia ${ }^{5}$, Francesco Picardo $^{2}$, Teresa Balsamo ${ }^{1}$, \\ Gerardo Paolo Flammia ${ }^{8}$, Domenico Trombetta ${ }^{1}$, Angela Pantalone ${ }^{2}$, Klaas Kok ${ }^{9}$, \\ Ferronika Paranita9, Lucia Anna Muscarella, ${ }^{1, *}$, Vito Michele Fazio, ${ }^{1,2, *}$ \\ ${ }^{1}$ Laboratory of Oncology, IRCCS "Casa Sollievo della Sofferenza" Hospital, San Giovanni Rotondo, Italy \\ ${ }^{2}$ Genetic and Clinic Pathology Unit, University Campus Bio-Medico of Rome, Rome, Italy \\ ${ }^{3}$ Unit of Biostatistics, IRCCS "Casa Sollievo della Sofferenza" Hospital, San Giovanni Rotondo, Italy \\ ${ }^{4}$ Department of Bioscience, Biotechnology and Biopharmaceutics, University of Bari, Bari, Italy \\ ${ }^{5}$ Department of Urology, Regina Elena National Cancer Institute, Rome, Ital \\ ${ }^{6}$ Department of Pathology, Regina Elena National Cancer Institute, Rome, Italy \\ 7Unit of Pathology, IRCCS "Casa Sollievo della Sofferenza" Hospital, San Giovanni Rotondo, Italy \\ ${ }^{8}$ UOC of Urology, Campus Bio-Medico University Hospital, Rome, Italy \\ ${ }^{9}$ Department of Genetics, University of Groningen, University Medical Center Groningen, The Netherlands \\ *These authors equally contributed to this work
}

Correspondence to: Lucia Anna Muscarella, email: I.muscarella@operapadrepio.it

Keywords: $C C R C C, K E A P 1$, methylation, epigenetic biomarker, outcome

Received: June 13, $2016 \quad$ Accepted: December 27, $2016 \quad$ Published: January 04, 2017

\section{ABSTRACT}

The Keap1/Nrf2 pathway is a master regulator of the cellular redox state through the induction of several antioxidant defence genes implicated in chemotherapeutic drugs resistance of tumor cells. An increasing body of evidence supports a key role for Keap1/Nrf2 pathway in kidney diseases and renal cell carcinoma (RCC), but data concerning the molecular basis and the clinical effect of its deregulation remain incomplete.

Here we present a molecular profiling of the KEAP1 and NFE2L2 genes in five different Renal Cell Carcinoma histotypes by analysing 89 tumor/normal paired tissues (clear cell Renal Carcinoma, ccRCCs; Oncocytomas; Papillary Renal Cell Carcinoma Type 1, PRCC1; Papillary Renal Cell Carcinoma Type 2, PRCC2; and Chromophobe Cell Carcinoma).

A tumor-specific DNA methylation of the KEAP1 gene promoter region was found as a specific feature of the cCRCC subtype $(18 / 37,48.6 \%)$ and a direct correlation with mRNA levels was confirmed by in vitro 5-azacytidine treatment. Analysis of an independent data set of 481 cCRCC and 265 PRCC tumors corroborates our results and multivariate analysis reveals a significant correlation among CCRCCs epigenetic KEAP1 silencing and staging, grading and overall survival.

Our molecular results show for the the first time the epigenetic silencing of KEAP1 promoter as the leading mechanism for modulation of KEAP1 expression in CCRCCs and corroborate the driver role of Keap1/Nrf2 axis deregulation with potential new function as independent epigenetic prognostic marker in renal cell carcinoma. 


\section{INTRODUCTION}

Renal cell carcinoma is the most common malignant neoplasm arising in the kidney and comprises an heterogeneous group of tumors. Various histotypes of RCC have come to be defined on the basis of their histologic appearance, the presence of distinct driver mutations, varying clinical course, and different responses to therapy $[1,2]$. On the basis of morphology RCCs are classified into clear cell carcinomas, papillary tumors, chromophobic tumors, oncocytomas, and collecting duct tumors. Clear cell Renal Cell Carcinoma (ccRCC) are the most common ones, with a frequency of $70-80 \%$ of all renal cancers. At time of diagnosis as many as one-third of the ccRCC patients may have a metastatic disease and about half of the patients will have a recurrence.

Papillary RCC, which accounts for $15 \%$ of RCCs encompasses type I papillary RCC (PRCC1) and type II papillary RCC (PRCC2). Although inhibition of the specific cellular signaling pathways has led to some clinical benefits, the effect is marginal and the prognosis remains poor for patients with advanced disease.

The premise that RCC histotypes might represent distinct diseases is underscored by multilevel genomicsbased taxonomy studies. Moreover, different DNA methylation patterns have been characterized both across and within the histology-based subgroups, revealing different RCC molecular subtypes, some of these in association with a more aggressive behaviour [3].

In both clear cell and papillary histotypes the Keap1/Nrf2 pathway, the KEAP1 (Kelch-like erythroidderived Cap-n-Collar Homology (ECH)-associated protein-1, NFE2L2 (Nuclear factor (erythroid-derived 2)-like2, and CUL3 (Cullin 3) were recently identified as probable drivers. This finding was consistent with the current knowledge that RCC belongs to the type of tumors in which the Nrf2 pathway was shown to be constitutively activated mainly by the loss of Keap 1 functions that lead to Nrf2 nuclear accumulation and enhances the transcription of Phase II enzymes [4]. The induced activation of metabolizing enzymes confers to neoplastic cells resistance to radio- and chemotherapies with growth and survival advantages during their transformation and progression [5-6]. Moreover, the transcription factor Nrf2 plays an important role from acute kidney injury to chronic kidney disease and cancer and transcriptional activity of Nrf2 has been inversely correlated with $\mathrm{FH}$ enzyme activity, which is loss in PRCC2 [7-8].

Genetic alterations of Keap1/Nrf2 axis were described with a variable incidence in RCC, more frequently in PRCC2 [3, 9]. Genetic alterations of the Keap1/Nrf2 pathway were reported in a very small fraction of ccRCC patients. However, several studies demonstrated a general high impact of $\mathrm{Nrf2}$ dysfunction in renal cell carcinoma, suggesting that the deregulation of the Keap1 may play a role in carcinogenesis process histotypes beside the presence of genomic alterations $[10,11]$. We have previously reported that epigenetic modification by promoter methylation is a main mechanism of regulation of KEAP1 gene expression in Non Small Cell Lung Cancer, malignant gliomas, breast cancer and that it was associated with worst progression free survival [12-16].

Since the role of DNA methylation and genes epigenetically altered in RCC have been an active area of research over the past decade, the main purpose of this study is to investigate the contribution of epigenetic deregulation of the KEAP1 gene in different histotypes of renal cell carcinomas.

To address this issue, the analyses were stratified for the main five histological subtypes of renal cancer: 37 ccRCCs, 15 PRCC1s, 13 PRCC2s, 14 Oncocytomas and 13 Chromophobe Renal Cancers.

A clear association of KEAP1 promoter methylation and the ccRCC histology was found in a training set of 37 cases with an incidence of $49 \%$. A direct effect on Keap1 mRNA levels was demonstrated by in vitro experiments on a set of four ccRCC cell lines. The specific correlation between the KEAPI methylation and the Clear Cell histology was also validated by using two independent datasets of $481 \mathrm{ccRCC}$ and 264 PRCC affected patients from The Cancer Genome Atlas (TCGA) portal, showing a significant correlation with the ccRCCs' staging, grading and overall survival.

DNA-based assays are often more robust than RNA-based assays, and genes inactivated by promoter hypermethylation may provide a better target for molecular screening strategies to identify targeted therapies. Since the histologic appearance is considered the primary determinant in the classification of Renal Cancer [17], the discovery of specific variations in methylation profiles could further help to stratify the clear cell renal carcinoma subtype from others [3]. Moreover, our findings of KEAP1 hypermethylation provide the first indication that this epigenetic mechanism is important also in the regulation of KEAP1 expression in an aggressive renal cancer histotype and could represents an additional and attractive diagnostic and prognostic biomarker.

\section{RESULTS}

\section{Patients and treatment}

Patients' clinico-pathological features of all the Renal Cell Carcinoma histotypes analyzed into the study are summarized in Table 1. Overall, the series included 89 patients grouped in: $37 \mathrm{ccRCCs}(41.6 \%), 15 \mathrm{PRCC} 1 \mathrm{~s}$ (16.9\%), 13 PRCC2s (11.2), 10 Chromophobe Renal Cell Carcinomas (14.6\%) and 14 Oncocytomas (15.7\%), respectively. The mean patient's age at the time of diagnosis was $63.0 \pm 13.5(\mathrm{yy} \pm \mathrm{SD})$ with a range from 23 to 86 years. More than half of the patients were men $(68.6 \%)$ versus $31.4 \%$ of women. All patients underwent 
Table 1: Clinical, tumor stages and histological features of RCC affected patients enrolled for the study $(n=89)$

\begin{tabular}{|c|c|c|}
\hline Characteristics & $n(\%)$ & ccRCC \\
\hline Age at the surgery (median (IQR)) & $63(23-86)$ & $56(48-70)$ \\
\hline Sex & 89 & 37 \\
\hline M & $61(68.6)$ & $28(76)$ \\
\hline $\mathrm{F}$ & $28(31.4)$ & $9(24)$ \\
\hline Histology & 89 & 37 \\
\hline Clear cell & $37(41.6)$ & \\
\hline Chromophobe & $13(14.6)$ & \\
\hline Papillary tipe 1 & $15(16.9)$ & \\
\hline Papillary type 2 & $10(11.2)$ & \\
\hline Oncocytoma & $14(15.7)$ & \\
\hline Tumour Stage (excluded oncocytomas) & $75(84.2)$ & \\
\hline $1 \mathrm{a}$ & $14(18,7)$ & $4(11)$ \\
\hline $1 \mathrm{~b}$ & $24(32)$ & $8(22)$ \\
\hline $2 \mathrm{a}$ & $9(12)$ & \\
\hline $2 \mathrm{~b}$ & $4(5.3)$ & $10(27)$ \\
\hline $3 a$ & $15(20)$ & $5(13)$ \\
\hline $3 b$ & $6(8)$ & $3(8)$ \\
\hline 4 & $3(4)$ & $0(0)$ \\
\hline Lymph nodes stage (excluded oncocytomas) & 75 & 30 \\
\hline N0 & $20(26.6)$ & $16(43)$ \\
\hline N1 & $1(1.4)$ & $0(0)$ \\
\hline $\mathrm{N} 2$ & $7(9.3)$ & $4(11)$ \\
\hline $\mathrm{Nx}$ & $47(62.7)$ & $17(46)$ \\
\hline Clinical Metastasis stage & 89 & 37 \\
\hline M0 & $76(85.3)$ & $16(43)$ \\
\hline M1 & $13(14.7)$ & $21(57)$ \\
\hline Fuhrman Grading (only ccRCC) & 37 & \\
\hline Grade I & $1(2.7)$ & \\
\hline Grade II & $5(13.5)$ & \\
\hline Grade III & $19(51.4)$ & \\
\hline Grade IV & $12(32.4)$ & \\
\hline PFS & 89 & 37 \\
\hline Yes & $26(29.2)$ & $16(43)$ \\
\hline No & $63(70.8)$ & $21(57)$ \\
\hline OS (excluded oncocytomas) & 75 & 37 \\
\hline Death & $7(9.3)$ & $3(8)$ \\
\hline Alive & $68(90.6)$ & $34(92)$ \\
\hline
\end{tabular}

Data are reported as median (IQR) for continuous variables and as frequencies and percentages for categorical variables; OS, Overall Survival.

curative surgery and all malignant lesions were subjected to staging according to the TNM system (2009 classification): we found $38 \mathrm{~T} 1$ cases $(50.7 \%), 13 \mathrm{~T} 2$ cases $(17.3 \%), 21$ $\mathrm{T} 3$ cases $(28 \%)$ and $3 \mathrm{~T} 4$ cases $(4 \%)$. According to the 
CAP guideline (College of American Pathologist) the Fuhrman grading was attributed only to ccRCC, showing a heterogeneous spectrum of aggressiveness: 1 case G1 (2.7\%), 5 G2 (13.5\%), 19 G3 (51.4\%) and 12 G4 (32.4\%).

Excluding benign lesions as oncocytomas, the lymph node involvement at the diagnosis was found only in 8 cases (N1 and N2 patients), whereas 67 patients didn't show lymphnode metastasis (Nx and N0 cases). During follow-up, 33 patients (37\%) showed a relapse with 7 cases of local/ renal recurrence (7.8\%) and 26 cases $(29.2 \%)$ of distant metastasis (target organs: lungs, bone, liver and brain). The mean and median follow-up time was respectively 31.2 and 24.5 months with a range of 1-108 months.

During follow-up, 9 patients (10.1\%) died of RCC, whereas $80(89.9 \%)$ patients were still alive, with $92.2 \%$ survived at last follow-up. An Overall Survival equal to $90.6 \%$ was observed. Clinical characteristics of our series are in line with the just published data [18-19]. The number of cases for each histotype was chosen similar to perform an effective comparison. The number of ccRCCs was larger than the others since the inclusion of the two different metastatic and non-metastatic groups.

\section{$K E A P 1$ promoter methylation profile in renal carcinoma tissues}

The KEAP1 methylation level was firstly evaluated on DNA obtained from a total of 89 RCC tissues, 70 paired normal renal tissues distant from the tumors (NRDT) and 10 normal renal tissues from patients affected by urothelial carcinoma (NR), (Supplementary Figures 2 and 3). The median values and Inter Quartile Ranges (IQR) for KEAP1/
$A C T B$ ratios were $0.0(0-0)$ for NR, 0 (0.000-0.329), for NRDT paired with ccRCC, and 0.611 (0.000-10.210) for ccRCC tumor tissues, (Figure 1). The discriminatory power of the KEAP1 QMSP assay was assessed by estimating the area under the ROC curve using paired normal renal tissues distant from tumor and ccRCCs. The AUC value was 0.68 , with an optimal cut-off value of 1.56 , a sensitivity of $49 \%$ and a specificity of $87 \%$. In ccRCC methylation was detected in 18 out of 37 cases $(48.6 \%)$, (Supplementary Figure 4). No statistically significant differences in methylation levels was detected between the NR and NRDT group. However, statistically significant differences were found when the NRDT and ccRCC group were compared ( $p=0.0054$; Wilcoxon Signed rank Test). No significant differences were found between NRDT and the other RCC histotypes.

In order to independently validate the specific correlation of data obtained to the ccRCC histotype, the $K E A P 1$ methylation and the functional effect of KEAP1 promoter methylation on its expression was analyzed in two independent datasets of $481 \mathrm{ccRCC}$ and 265 PRCCs cancer samples available from The Cancer Genome Atlas portal (TCGA), (for ccRCC cohort characteristics see additional Supplementary Table 2). The KEAP1 gene has a $1.2 \mathrm{~kb}$ CpG island ( chr19:10613047-10614280, hg19/GRCh37) extending from the promoter region to intron 1 , that is recognized by seven probes (denoted as 1 to 7) present on the Illumina Human-Methylation450 Bead Chip, all near the transcription start site of the KEAPl gene (Figure 2A).

A highly significant inverse correlation of aberrant KEAP1 promoter methylation and KEAP1 mRNA expression was found in both ccRCC and PRCC samples

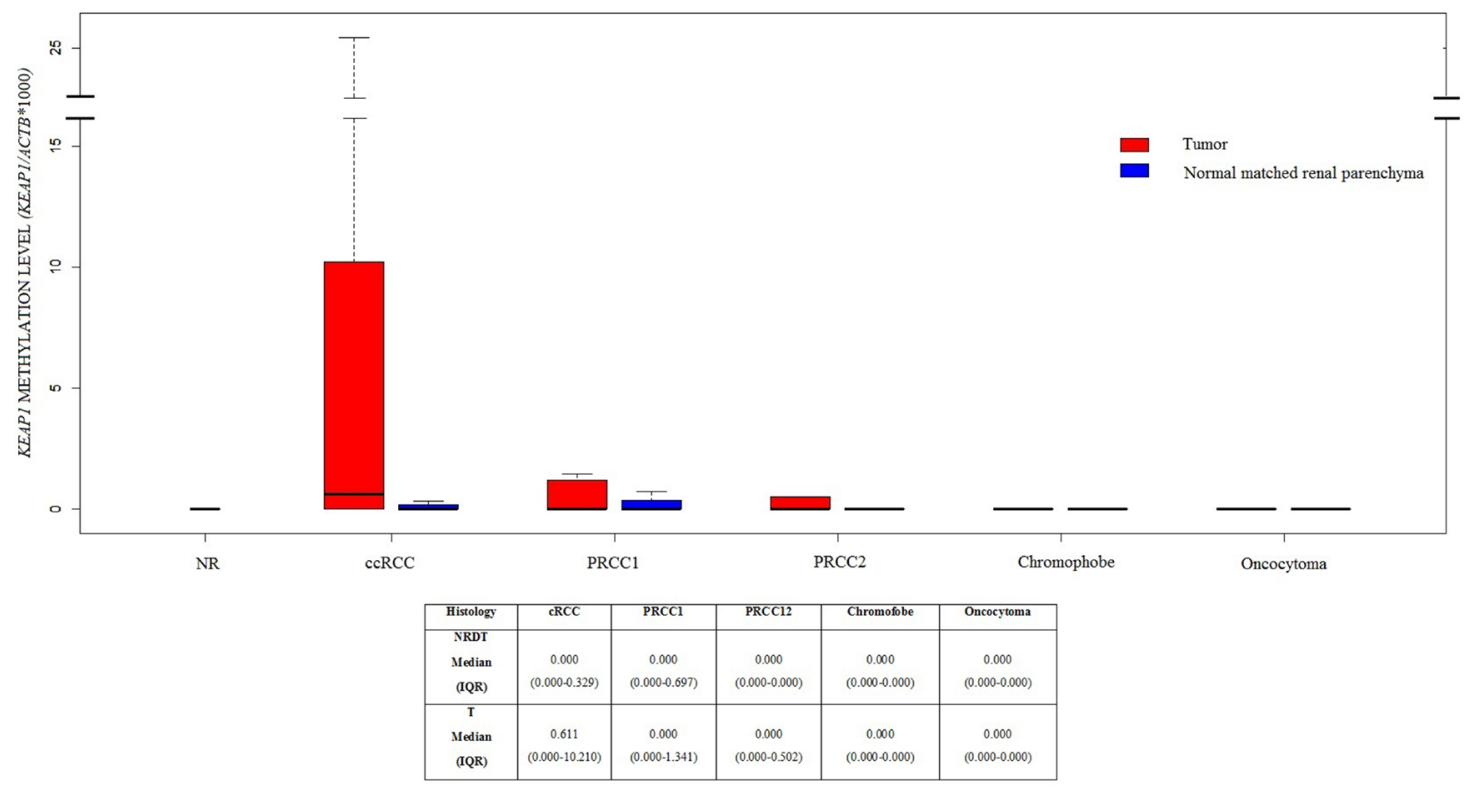

Figure 1: Boxplots of $K E A P 1$ promoter methylation levels in Normal Renal tissues (NR), and different renal tumor histologies included into this study, paired with normal renal parenchyma distant from tumor. Methylation levels are expressed as the $(K E A P 1 / A C T B)^{*} 1,000$. The boxes mark the interquartile range, (interval between the 25th and 75 th percentile). ccRCC, clear cell Renal Cell Cancer; PRCC1, Papillary Renal Cell Carcinoma Type 1; PRCC2, Papillary Renal Cell Carcinoma Type 2. 
showing a methylation value $>0,10$ (Figure $2 \mathrm{~B}$ ). However, a methylation value $>0,10$ was observed only in $3 \%$ (7 out of 265) of PRCCs, thus confirming results obtained on our small cohort of an inconsistent contribution of the $K E A P 1$ promoter epigenetic silencing in this histology, (Supplementary Figure 5).

\section{Association of $K E A P 1$ methylation with clinical parameters in ccRCCs}

We finally examined the correlation between the KEAP1 QMSP methylation level and patients' clinical data. Specifically, we did not find any significant correlation between the KEAP1 methylation level and lymph node status, or grading. Moreover, there was no significant association between KEAP1 methylation and disease free survival, local recurrence or overall survival. Taking into account the few samples available, the same correlation analysis was then performed on the TCGA ccRCC dataset. For each $\mathrm{CpG}$ site of the KEAPl promoter gene region, the samples were stratified according KEAPl methylation median level to estimate the association with clinical parameters as independent prognostic molecular marker.
Relevant results were summarized in Table 2: $\mathrm{CpG}$ methylation at exon 1 and intron 1 revealed significant association with Overall Survival (OS), grading, staging and tumor dimension. No association was found with age and sex, lymphonodes count and metastasis.

\section{Restoration of KEAP1 expression correlated with demethylation by 5-aza-dC treatment}

The methylation status of the four $c c R C C$ cell lines $F G$-2, $F W, 5$ and $E W$ was assessed. QMSP analysis showed a variable methylation level of KEAP1 (Figure 3). To verify whether the repression of KEAP1 expression was correlated with $\mathrm{CpG}$ methylation in the gene promoter, we examined the variation of KEAP1 mRNA level in the four cell lines before and during treatment with 5-aza-dC (Figure 4A, 4C, 4E). By real-time quantitative PCR analysis a progressive increase in the KEAP1 transcript abundance was observed after $48 \mathrm{~h}(p<0.0001)$ and was shown to correlate in ccRCC FW, ccRCC FG-2, ccRCC 5 with a decreased KEAP1 promoter methylation at $48 \mathrm{~h}(p<0.001$ respectively), (Figure 4B, 4D, 4F). Keap1 expression did not reveal any significant variation in the ccRCC EW.

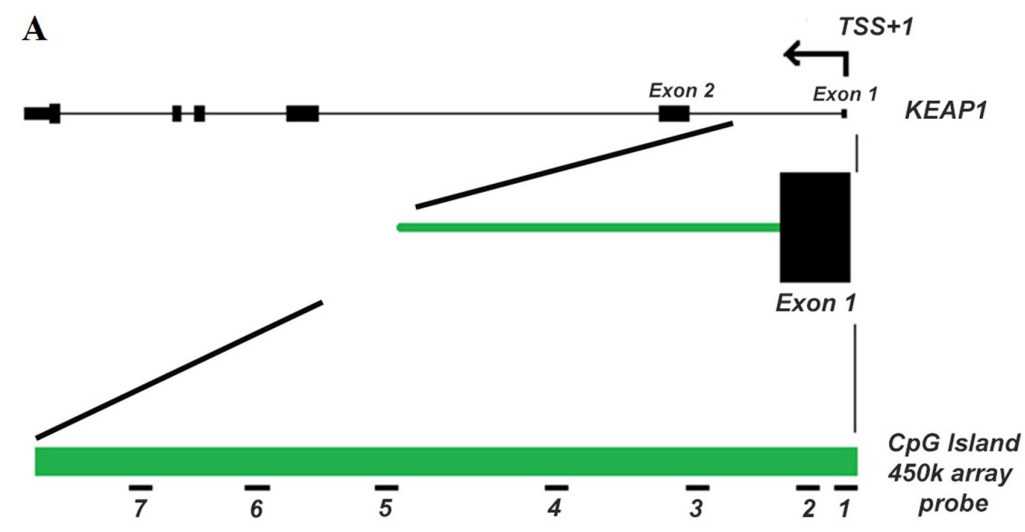

B

\begin{tabular}{|c|c|c|c|}
\hline CpG & KEAP1 gene region & Spearman rho correlation & $p$ value \\
\hline $\operatorname{cg} 06911149$ & Exon 1 & -0.20 & $<.0001$ \\
\hline $\operatorname{cg} 02428100$ & Exon 1 & -0.24 & $<.0001$ \\
\hline $\operatorname{cg} 03890664$ & Intron 1 & -0.10 & 0.0165 \\
\hline $\operatorname{cg} 15204119$ & Intron 1 & -0.25 & $<.0001$ \\
\hline $\operatorname{cg} 15676203$ & Intron 1 & -0.19 & $<.0001$ \\
\hline $\operatorname{cg} 26500801$ & Intron 1 & -0.26 & $<.0001$ \\
\hline $\operatorname{cg} 26988016$ & Intron 1 & -0.21 & $<.0001$ \\
\hline
\end{tabular}

Figure 2: The $450 \mathrm{~K}$ methylation array data from the TCGA demonstrates that methylation of the KEAP1 promoter region is a hallmark in ccRCC. (A) The KEAPl gene structure, including its transcription start site (TSS), exons (black boxes) and introns (lines), a 1.2-kb CpG island of the KEAPl gene (green bar) and the location of the probes present on the $450 \mathrm{~K}$ array (short black numbered lines, denoted as probes 1-7 for target ID cg06911149 and cg02428100 located into the exon 1 and $\operatorname{cg} 03890664$, cg15204119, cg15676203, cg26500801, cg26988016 located into the intron 1). (B) Inverse correlation of KEAP1 mRNA expression with the DNA methylation status in ccRCC. p: Spearman correlation coefficient. 
Table 2: Prognostic association of CpGs located into the KEAP1 promoter region with clinical

\begin{tabular}{ccccccc}
\hline CpG Island & $\begin{array}{c}\text { KEAP1 gene } \\
\text { location }\end{array}$ & OS & $\begin{array}{c}\text { Fuhrman } \\
\text { Grade }\end{array}$ & Staging & T & $\begin{array}{c}\text { KEAP1 expression } \\
(\boldsymbol{r}, \boldsymbol{p} \text { value })\end{array}$ \\
\hline $\operatorname{cg} 06911149$ & exon 1 & 0.01 & 0.0107 & 0.0005 & 0.0003 & $-0.20,<.0001$ \\
$\operatorname{cg} 02428100$ & exon 1 & 0.426 & 0.0287 & 0.027 & 0.008 & $-0.24,<.0001$ \\
$\operatorname{cg} 03890664$ & intron 1 & 0.139 & 0.9084 & 0.1388 & 0.2529 & $-0.10,0,0165$ \\
$\operatorname{cg} 15204119$ & intron 1 & 0.002 & 0.0019 & $<00001$ & 0.0015 & $-0.25,<.0001$ \\
$\operatorname{cg} 15676203$ & intron 1 & 0.009 & 0.0037 & 0.0007 & 0.0005 & $-0.19,<.0001$ \\
$\operatorname{cg} 26500801$ & intron 1 & 0.013 & 0.0549 & 0.0126 & 0.0183 & $-0.25,<.0001$ \\
$\operatorname{cg} 26988016$ & intron 1 & $<0.001$ & 0.5512 & 0.0399 & 0.0147 & $-0.21,<.0001$ \\
\hline
\end{tabular}

characteristics and Overall Survival.

KEAP1 deregulation is not caused by somatic alterations in the DGR domain of $K E A P 1$ or Nhe2 domain of NFE2L2 in renal cell carcinoma

An alternative mechanism to achieve the deregulation of KEAP1 would be the occurrence of somatic mutations in our RCC samples. To exclude this possibility we analysed 20 ccRCCs (11 KEAP1 methylated and 9 unmethylated samples) and 10 PRCC2 tumors for somatic alterations in the most frequently mutated coding regions of the KEAP1 and NFE2L2 genes. Specifically, we search for molecular lesions in the KEAP1 Double Glycin Region (DGR) domain, which contains binding sites for Nrf2, $\mathrm{Bcl} 2$, Pgam5 and Ikk $\beta$ proteins. We also check for somatic alterations in the Nhe2 domain of the NFE2L2. This analysis did not reveal any sequence variations that would likely result in a functional alteration in Keap1 or Nrf2 proteins, (Supplementary Table 2).

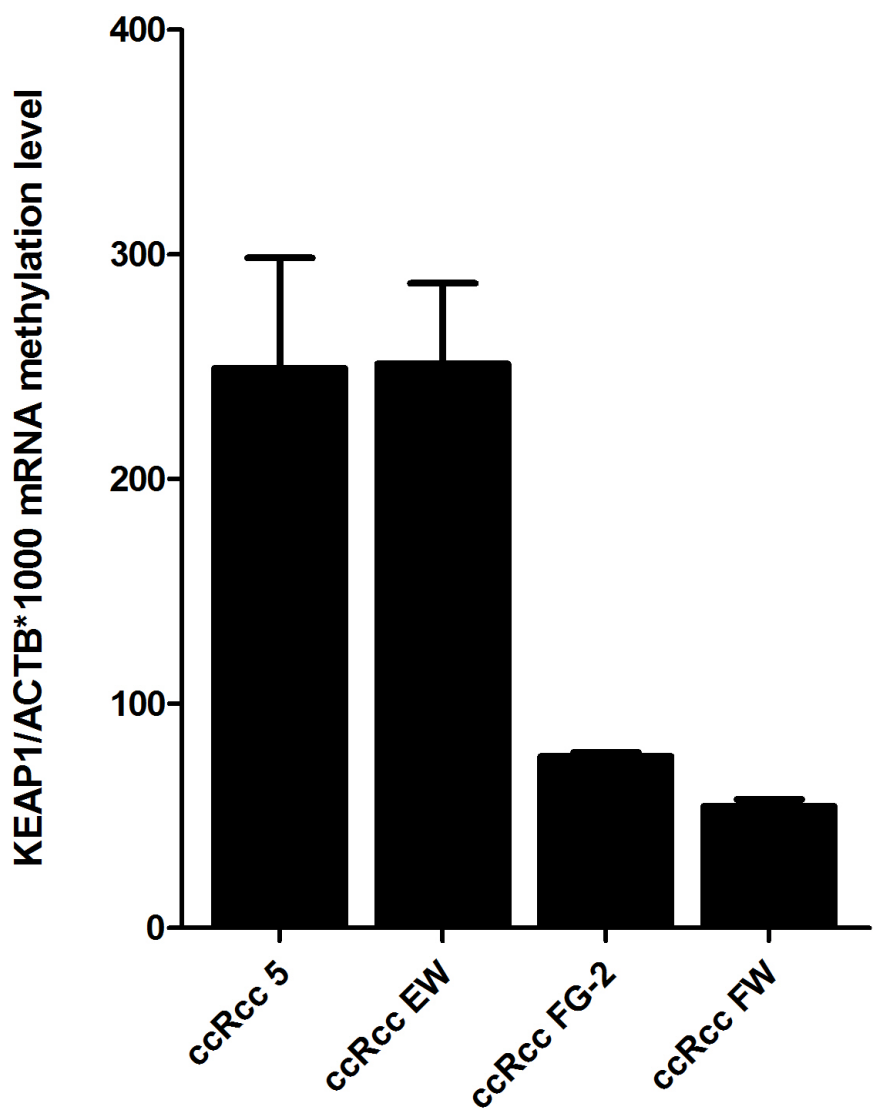

Figure 3: KEAP1 promoter methylation levels in four ccRCC cell lines $(5, \mathrm{EW}, \mathrm{FG}-2, \mathrm{FW})$ detected by using quantitative methylation real-time PCR. Values were reported as the KEAPI/ACTB ratio*1000. Error bars indicate the standard error of three different experiments. 


\section{$K E A P 1$ promoter methylation and Keap1 immunostaining}

To assess the potential correlation between the Keap1 protein levels in ccRCCs and the epigenetic silencing of the KEAP1 gene, 15 ccRCC tumor cases showing KEAP1 promoter methylation and 14 tumors without methylation were analysed by immunohistochemical analysis.
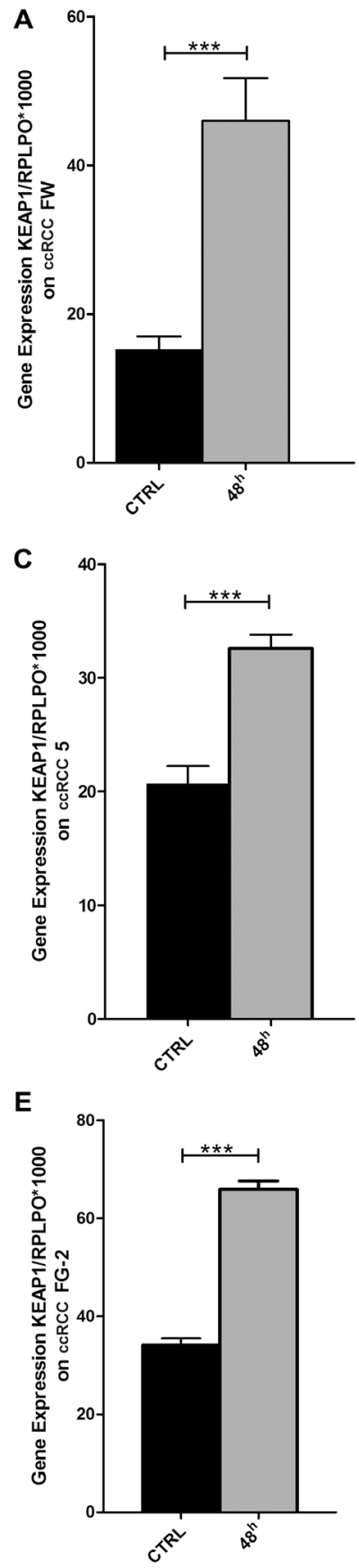

A semi-quantitative scoring system based on staining intensity and percentage of positive neoplastic cells to evaluate Keap1 immunoreactivity was applied. All the areas of the tissue section were evaluated for Keap1 protein expression. Cases were scored as positive if independently evaluated by two pathologists (PG and PP). Keap1 immunoreactivity was observed in the cytoplasm of tumor cells. Representative images of
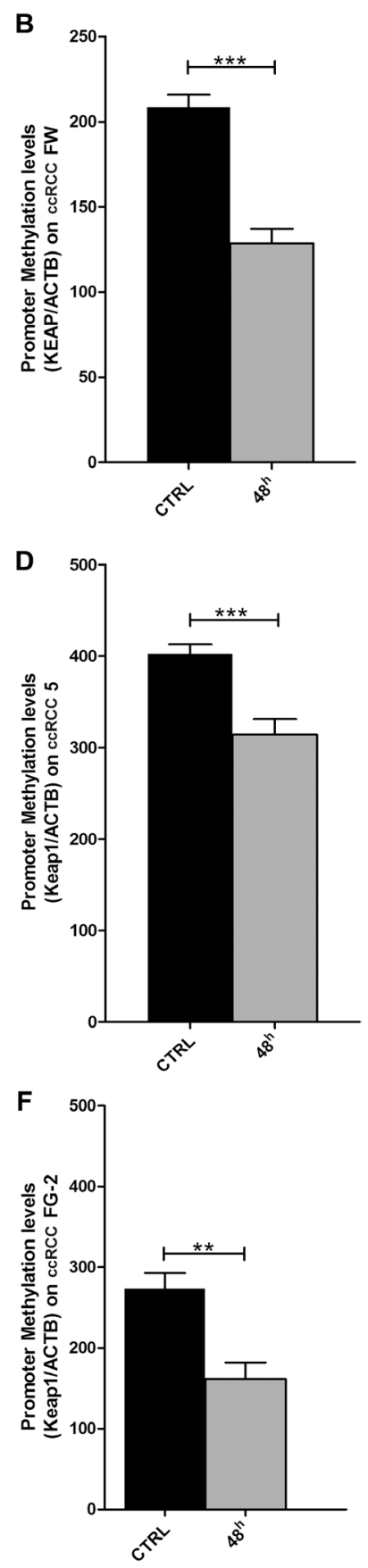

Figure 4: Changes in KEAP1 mRNA transcript levels in the (A) ccRCC-FW, (C) ccRCC 5, (E) ccRCC FG-2 cell lines by quantitative real-time RT-PCR before (CTRL) and after treatment with $5 \mu \mathrm{m}$ of 5-azacytidine at 48 hours (AZA $48 \mathrm{~h}$ ). Error bars indicate the standard deviation of three different experiments. Changes in KEAP1 promoter methylation levels in the (B) ccRCC FW, (D) ccRCC 5, (F) ccRCC FG-2 cell lines by quantitative methylation real-time PCR before (CTRL) and after treatment with $5 \mu$ m of 5 -azacytidine at 48 hours (AZA $48 \mathrm{~h}$ ). Error bars indicate the standard deviation of three different experiments. ${ }^{*} p<0.01, * * * p<0.001$. 
immunohistochemical staining are shown in Figure 5. A no statistically significant difference was observed.

\section{DISCUSSION}

A growing amount of evidence suggest that an increased activity of Nrf2 due to NFE2L2 or KEAP1 mutations may play a pivotal role in the pathogenesis of many solid tumors and recently emerged as one of the main pathways implicated in Renal Carcinoma [13, 20-22]. Of note, deregulation of Keap1/Nrf2 pathway was highlighted in the Papillary Type 2 subtype of renal cancer as a consequence of an abnormal fumarate accumulation in congenital fumarate hydratase deficiency $[13,20]$ which predisposes to PRCC2.

Somatic mutations in NFE2L2 and CUL3 genes were also frequently reported in sporadic cases with PRCC2, but less frequently for the clear-cell subtypes of RCC. Somatic mutations in NFE2L2 and CUL3 genes were also reported more frequently for sporadic cases with PRCC2 than for the clear-cell subtypes of RCC [21, 23].

This could be explained taking into account the heterogeneity features of renal cancer and more importantly by an existing alternative epigenetic mechanism to regulate Keap1-Nrf2 signalling. KEAP1 promoter epigenetic silencing as just reported in cancers as well as in the other diseases with a clear association with tumors having a low incidence of somatic mutations [22, 24].

Since no data are available regarding the contribution of KEAP1 hypermethylation in renal cancer, we decided to investigate this epigenetic mechanism of silencing by performing a comprehensive genetic and epigenetic analysis in 89 surgical resected RCCs. As main result, an aberrant promoter KEAP1 methylation was identified in the ccRCC subset with a frequency of $49 \%$. The strong correlations with this specific histotype was then confirmed by the revision of the TCGA data for two different cohorts of $481 \mathrm{ccRCCs}$ and 265 PRCCs. Moreover, we demonstrated that the methylation of the KEAPI CpG island represents a critical mechanism regulating the transcription levels of the KEAP1 gene by showing a statistical significant inverse Pearson Correlation between KEAP1 methylation and transcript levels. Further, these data are corroborated by the fact that pharmacological 5-Aza treatment is able to restore the expression of Keap1 in 3 out of 4 different ccRCC cell lines.

Methylation levels did not correlate with the Keap1 protein expression assessed in 29 ccRCC tumors by IHC analysis. The reasons for this lack of association might be due to several reasons. First, there is a lack of a consistently defined cut-off value for the semiquantitative immunohistochemical scoring and to detection limit [25]. Moreover, ccRCCs are heterogeneous/multifocal tumours and intra-tumoral heterogeneity could involve epigenetic changes similar to genetic and genomic events [26-27]. QMSP is a highly sensitive methodology and methylation signals may be obtained even if cells that carry KEAP1 promoter methylation represent a small proportion among the majority of cells with unmethylated promoter. Conversely, IHC may not be able to detect small clusters of cells that have lost protein expression."

No genetic abnormalities were identified by analysing the DNA obtained from a subset of $20 \mathrm{ccRCCs}$ and 14 PRCC2s. This result is in line with public data on the somatic mutation frequency of KEAP1 in RCC. The COSMIC database has KEAPI sequence data on 953 cases of ccRCC of which only 8 had a nonsynonymous KEAP1 mutation (0.8\%) and 1/875 had a non-synonymous NFE2L2 mutation (0.1\%). Moreover, in the TCGA analysed dataset, only 2 out of $446 \mathrm{ccRCCs}$ had a mutations $(0.5 \%)$. In papillary RCCs, 8 out of 183 non-synonymous NFE2L2 mutations are described (4\%), [28]. This result partially confirms the previous available

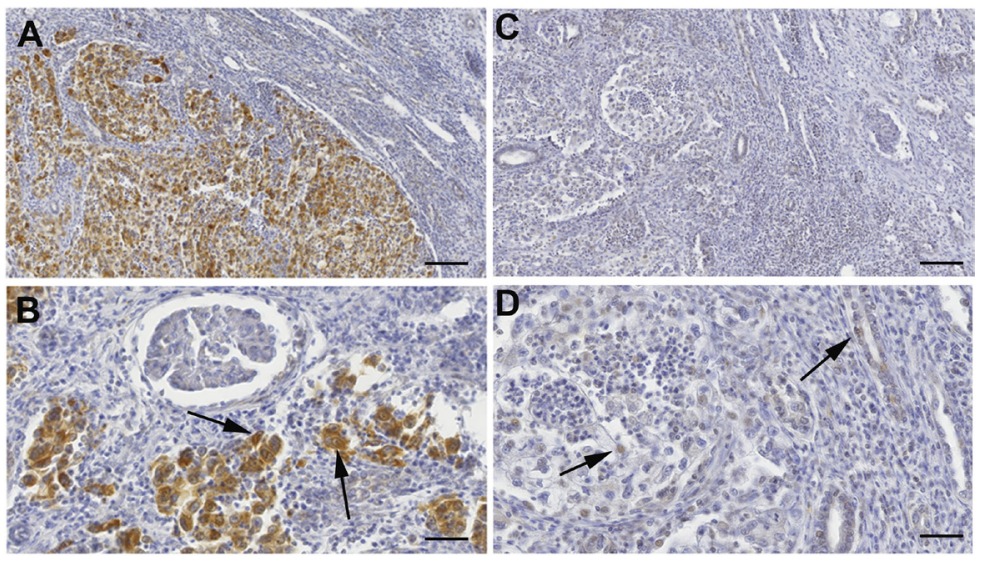

Figure 5: Keap1 protein expression by immunohistochemical analysis. (A-B) Microphotographs are from a ccRCC sample negative for KEAP1 promoter methylation. (A) Original magnification X9. Scale bar $110 \mu \mathrm{m}$; (B) Original magnification X25. Scale bar $40 \mu \mathrm{m}$. Tissue-associated macrophages are positive for Keap1 staining and are used as internal positive controls. (C-D) Weak staining in one ccRCC case with KEAP1 promoter methylation. (C) Original magnification X9. Scale bar $110 \mu$ m; (D) Original magnification X25. Scale bar $40 \mu \mathrm{m}$. Tissue-associated macrophages are positive for Keap1 staining and are used as internal positive controls. 
published data that reported a low rate of inactivating mutations of CUL3 or activating mutations of NFE2L2 in sporadic PRCC2.

Deregulation of the Nrf2/Keap1 system has been linked to patient's outcome and to resistance to a variety of anticancer drugs, due to the key role of Nrf2 in modulating the expression of phase II drug metabolism enzymes and phase III drug transporters [17]. In patients affected by solid tumors, a nuclear Nrf2 accumulation in combination with a low or absent Keap1 expression was associated with poor outcome. However, this was not well investigated in Renal Cancer. In our study follow up data were available for all 37 ccRCC cases. No evidence of a statistical significant correlation with the risk to progress was observed for patients bearing epigenetic abnormalities. This may be mainly due to the statistical power of the small sample cohort. Therefore, we extended our studies by analysing $450 \mathrm{~K}$ methylation array data for the $\mathrm{CpG}$ promoter of $K E A P 1$ for an independent cohort of 481 ccRCC patients with available clinic-pathological data. A clear association was observed between the hypermethylation of $\mathrm{CpGs}$ located in the KEAP1 promoter and an increased ccRCC tumor grading and staging, further supporting their biological relevance. Furthermore, the data analysis suggests that KEAPl hypermethylation is able to predict patients' survival. An increased methylation level of 5/7 CpGs was strongly associated with worse OS in ccRCCs.

Our study reports for the first time the epigenetic modulation of KEAP1 by CpGs promoter hypermethylation as the leading mechanism of KEAP1 deregulation in ccRCC and together with recently published data on PRCC2, corroborate the hypothesis of a driver role of the Keap1/Nrf2 pathway in the ccRCCs subtypes with a specific epigenetic deregulation mechanism. In line with data from previous molecular studies, this epigenetic finding represents a new disease molecular marker for a specific subtype of RCC. An important avenue of future work will be to complement this epigenetic biomarker to existing renal cancer genetic biomarkers to assess their importance from the standpoint of therapy and clarify their potential for application in the clinical setting. At the same time, based on the high frequency of tumors displaying aberrant Nrf2 activation, Nrf2 should be regarded as an important new pharmacological target [29, 30].

\section{MATERIALS AND METHODS}

\section{Cell lines}

ccRCC FG-2, ccRCC FW, ccRCC 5 and ccRCC EW clear renal cancer cell lines were obtained in collaboration with the UMCG, Department of Genetics, Groningen, the Netherland (Dr. Klaas Kok, PhD) and were cultivated in RPMI 1640 medium, supplemented with 10\% FBS.
DNA was extracted from each cell line by using the standard procedure with Phenol-Chloroform. RNA was extracted using Trizol reagent (Life Technologies) in according to the manufacturer's instructions. DNA and RNA concentrations were estimated by NanoDrop Spectrophotometer ND-1000 (Thermo Scientific).

\section{Patients and tissue samples}

The patients' clinical and pathological data including pathological TNM staging, site of the lesion, grading, age, gender, and follow-up data were collected and are shown in Table 1.

Renal cancer tissues samples were obtained as Formalin Fixed Paraffin Embedded (FFPE) specimens from 89 patients surgically treated in the Department of Urology at the "Regina Elena" National Cancer Institute of Rome. All tissue specimens showing features of Clear Renal Cell Carcinoma (ccRCC, $n=37$ ), Type I Papillary Renal Cell Carcinoma (PRCC1, $n=15$ ), Type II Papillary Renal Cell Carcinoma (PRCC2, $n=13$ ), Oncocytoma $(n=14)$ and Chromophobe Renal Cell Carcinoma ( $n=13)$, attending the Outpatient Clinic of the Department of Urology or undergoing to curative surgical treatment during the period 2003-2013. Wherever possible, all available paired histologically confirmed normal renal tissues distant from tumor (NRDT, $n=70$ ) and normal renal tissue samples from urothelial tumor patients (NR, $n=10)$ were collected. All human materials used in the study were obtained in compliance with guidelines of the Local Ethical Committee.

After excision, tissues were collected in $10 \%$ formalin and embedded in paraffin for histopathological and immunohistochemical analysis. Tumor samples with at least $70 \%$ cancer cells were eligible for direct genetic and epigenetic analyses. Cases showing tumor cellularity lower than $70 \%$ were microdissected for enrichment of neoplastic cell content.

\section{DNA extraction}

Sections, 3- $\mu$ m-thick, were cut from FFPE tissue blocks and subjected to Hematoxilyn and Eosin (H\&E) staining to verify tumor cellularity. Under light microscope, 12 - $\mu$ m-thick sections of tumor specimens were then carefully dissected to enrich for areas that contained tumor cells. DNA was subsequently extracted from the paraffinembedded sections as previously described [31]. DNA concentrations were measured by using Thermo Scientific NanoDrop $^{\mathrm{TM}} 1000$ Spectrophotometer (Thermo Scientific).

\section{Mutation analysis}

DNA obtained from $20 \mathrm{ccRCC}$ and 10 PRCC2 tissues was analysed by fluorescence-based direct sequencing of the entire KEAPl gene region encoding the DGR (Double 
Glycine Repeat) domain of the Keap1 protein and exon 2 of the NFE2LE gene, encoding the Nhe2 domain (Supplementary Table 3). Amplification reactions were performed by using the Gene Amp PCR System 9700 Thermal cycler (Applied Biosystem, Foster City, CA), in a final reaction volume of $25 \mu \mathrm{l}$ containing $100 \mathrm{ng}$ of genomic DNA template, $0.25 \mathrm{nM}$ dNTPs, 20 pmol of each primers, $1 \mathrm{U}$ HotMaster Taq polymerase (Eppendorf AG, Hamburg, Germany), in 10X Hot Master Taq Buffer with magnesium and sterile distilled water. PCR cycling conditions include an initial denaturation step at $94^{\circ} \mathrm{C}$ for $2 \mathrm{~min}$, followed by 35 cycles of $94^{\circ} \mathrm{C}$ for $30 \mathrm{sec}$, annealing for $30 \mathrm{sec}$, extension at $72^{\circ} \mathrm{C}$ for $30 \mathrm{sec}$ and ending with a final elongation step at $72^{\circ} \mathrm{C}$ for $7 \mathrm{~min}$. PCR products were purified using GFX PCR DNA and the Gel Band Purification Kit (GE Healthcare, Buckinghamshire, UK) and sequenced by using the Big Dye Terminator Ready Reaction mix v. 1.1 on an ABI 3100 sequence detection system with the Sequencing Analysis software v.3.7 (Applied Biosystems).

\section{Bisulfite conversion of DNA and quantitative methylation specific PCR analysis (QMSP)}

One microgram of DNA extracted from cell lines and tissue samples was subjected to bisulfite treatment and DNA purification using the Epitect Bisulphite kit (Qiagen Sci, MD, USA) according to manufacturer's instruction. Bisulfitemodified DNA was used as template for Quantitative Methylation Specific PCR (qMS-PCR) to detect converted DNA. Primer/probe sets for the KEAPl promoter region and for the unmethylated promoter region of the $A C T B$ as reference gene were previously described and are reported in Supplementary Table 1 [12]. Calibration curves for both target and reference genes were constructed using serial dilutions (90-0.009 ng) of commercially available fully methylated DNA (CpGenome Universal Methylated DNA, Millipore, Chemicon, cat\#S7821). Amplification reactions were carried out in triplicate in 384-well plates and in a volume of $10 \mathrm{~mL}$ that contained $50 \mathrm{ng}$ of bisulfite-modified DNA on a ABI PRISM 7900 Sequence detection system and were analysed by SDS 2.1.1 software (Thermo Fisher Inc., Applied Biosystems division). Each plate included calibration curves for the $A C T B$ and KEAPl genes, patients' DNA samples, a positive control CpGenome Universal Methylated DNA, and multiple water blanks (Supplementary Figure 1). The Cp (cross point) values of each QMSP reaction were calculated using the second derivative maximum method. The qMS-PCR standard curves of the KEAP1 and ACTB genes for the normalization of the input DNA were established with CpGenome Universal Methylated DNA. Methylation levels were finally calculated as the ratio of KEAP1 to $A C T B$ and then multiplied by 1000 for easier tabulation (average value of triplicates of Target Gene/average value of triplicates of $A C T B \times 1000$ ).

\section{In vitro 5-Aza-2'-deoxycytidine (5-aza-dC) treatment}

FG-2, FW, 5 and EW ccRCC cell lines were seeded in a 6 well dish. The 5-aza-2'-deoxycytidine (DAC), an inhibitor of DNA methyltransferase, was added in a concentration of $5 \mu \mathrm{M}$ (Sigma-Aldrich) with fresh medium for $24 \mathrm{~h}$ and $48 \mathrm{~h}$. At both time points $(24 \mathrm{~h}$ and $48 \mathrm{~h}$ ) cells were harvested for DNA and RNA isolation to interrogate the effects of induced DNA demethylation and analyse the KEAP1 expression levels.

\section{Quantification of the KEAP1 expression by real-time PCR}

PCR fragments for KEAPI and RPLPO were amplified by the Taqman assay listed in Supplementary Table 1, and were cloned into the StrataClone ${ }^{\mathrm{TM}}$ PCR Cloning Vector pSC-A (Stratagene, Milan, Italy). Miniprep cultures were grown in $5 \mathrm{ml}$ of LB-Ampicillin broth. Plasmid DNA from the selected transformed cells was isolated using the QIAprep ${ }^{\circledR}$ Spin Miniprep Kit (Qiagen) Five plasmid dilutions in the range of $1 \times 10^{6}$ copies to $1 \times 10^{2}$ copies were used to construct the standard curves for real-time PCR.

First strand cDNA synthesis from $1 \mu \mathrm{g}$ of total RNA extracted from renal cell lines was carried out with SuperScript III First-Strand Synthesis (Thermo Fisher, Invitrogen Division, Carlsbad, CA, USA) using a gene expression amplification mixture containing $2.5 \times$ TaqMan $^{\circledR}$ Universal PCR Master Mix (Thermo Fisher, Life Technologies division), $250 \mathrm{nM}$ of TaqMan ${ }^{\mathrm{TM}}$ Gene Expression Assay with TaqMan probe and $1 \mu \mathrm{l}$ of template cDNA or plasmid product (serial dilutions), (Table 1). Reactions were run on ABIPRISM 7900HT Sequence Detection System (Thermo Fisher, Life Technologies division). Protocol conditions were as follows: $10 \mathrm{~min}$ at $95^{\circ} \mathrm{C}, 40$ cycles at $95^{\circ} \mathrm{C}$ for $15 \mathrm{~s}$ and $60^{\circ} \mathrm{C}$ for $60 \mathrm{~s}$. Each assay was carried out in triplicate and the transcription level was normalized using $R P L P O$ as reference gene. Calibration curves for the KEAPI and PRLPO genes (used as calculation method) were constructed and sample concentration was calculated using the plasmid standard curve, resulting in plasmid concentrations expressed as copy number of corresponding standard molecules. The relative sample amount was expressed as ratio marker ([KEAP1/RPLPO $]^{*} 1000$ for an easier tabulation).

\section{Immunohistochemistry (IHC)}

Formalin fixed paraffin-embedded sections $(3 \mu \mathrm{m})$ of 29 ccRCCs were selected for IHC analysis and collected on polarized slides. The sections were deparaffinised in xylene, hydrated in gradient alcohol, and warmed in TrisEDTA buffer $(0.01 \mathrm{M}, \mathrm{pH}=9.0)$ for antigen retrieval at 
$98^{\circ} \mathrm{C}$. The sections were then incubated with hydrogen peroxide $(0.3 \% \mathrm{v} / \mathrm{v})$ in methanol for $5 \mathrm{~min}$ to quench the endogenous peroxidase activity. Thereafter, the slides were incubated with rabbit polyclonal anti-Keap1 antibody (AP-20503, Proteintech, Chicago, USA) for $60 \mathrm{~min}$ at RT. The primary antibody was detected by using commercially available detection kit (EnVision ${ }^{\mathrm{TM}} \mathrm{FLEX}+$, Dako, Glostrup, Denmark) following the manufacturer's protocol and diaminobenzidine as chromogen.

Slides were washed with Tris-buffered saline (TBS, $0.1 \mathrm{M}, \mathrm{pH}=7.4), 3-5$ times after each step. Finally, the sections were counterstained with Mayer's hematoxylin and mounted with Biomount (BIO-OPTICA, Milan, Italy). In the negative control tissue sections, the primary antibody was replaced by isotype specific non-immune rabbit IgG. Tissue sections from colon cancer were used as a positive control for Keaplexpression. The sections were evaluated by light microscopic examination on Olympus BX51 microscope.

Each slide was evaluated for Keap1 immunostaining by using a semi-quantitative scoring system combining staining intensity and the percentage of positive neoplastic cells.

Immunoreactivity was assessed in all the areas of the tissue section. Keap1 protein expression, independently evaluated by two Pathologists (PG and PP) was scored as positive if cytoplasm reactivity was observed in tumor cells. The semi-quantitative scale based on the $\%$ of immunoreactive neoplastic cells was: $0-10 \%=0$; $10-30 \%=1 ; 30-50 \%=2 ; 50-70 \%=3$ and $70-100 \%=4$. Sections were also scored on the basis of staining intensity as negative $=0 ;$ mild $=1 ;$ moderate $=2 ;$ intense $=3$. Finally, a immunoreactive staining score (IRS) was obtained by adding the score of percentage positivity and intensity and samples were classified ad negative/weak staining $($ IRS $<2$ ) and moderated/strong staining (IRS $\geq 2$ ).

\section{Validation of the $K E A P 1$ expression and promoter hypermethylation in two independent set of ccRCC and PRCC tumors (TCGA data analysis)}

KEAP1 Methylation and mRNA expression data for ccRCCs and PRCCs were downloaded from the "The Cancer Genome Atlas" (TCGA) data portal (https://tcga-data.nci.nih. gov). These data include $n=481$ ccRCCs and $n=265$ PRCC affected patients from two independent platforms: Illumina Infinium DNA methylation (HumanMethylation $450 \mathrm{~K}$ ) and Illumina HiSeq gene expression arrays.

\section{Statistical data analysis}

All analyses were performed using SAS Release 9.3 (SAS Institute, Cary, NC). A $p$ value $<0.05$ was considered for statistical significance.

Patients baseline characteristics were reported as mean and standard deviation or median and interquartile range and frequencies and percentages for continuous and categorical variables, respectively. Baseline comparisons were performed using Mann-Whitney $U$-test or Pearson chi-squared test for continuous and categorical variables, respectively.

Methylation level comparison between tumors and paired normal tissues was performed by using the Wilcoxon Signed Rank test. ROC curve analysis was performed to assess the diagnostic performances of KEAP1 methylation levels. Sensitivity and specificity were also reported at the optimal cut-off which maximized jointly sensitivity and specificity.

Correlation between KEAP1 mRNA expression (TCGA Illumina HiSeq platform) and the level of KEAP1 methylation was assessed using Spearman correlation coefficient.

Association between IHC and methylation was tested using Mc Nemar test.

Proportional hazard Cox regression analysis was performed to assess the association between KEAP1 methylation and overall survival.

For in vitro experiments, group comparisons were performed using two-tailed Student's $t$-test.

\section{CONFLICTS OF INTEREST}

The authors declare that they have no competing financial interests.

\section{FINANCIAL SUPPORT}

This work was supported by the Italian Ministry of Health (Ricerca Corrente RC1503LO51 and GR program 2010-2316264 to L.A.M.), by the "5 × 1000" voluntary contributions and by the AIRC/MFAG grant 12983 (to L.A.M.); by UCBM project "PEI0031_37351-14" (to VMF) and AIRC/MFAG grant 10520 (to MLP).

\section{REFERENCES}

1 Cancer Genome Atlas Research Network. Comprehensive molecular characterization of clear cell renal cell carcinoma. Nature. 2016; 499:43-49.

2. Davis CF, Ricketts CJ, Wang M, Yang L, Cherniack AD, Shen H, Buhay C, Kang H, Kim SC, Fahey CC, Hacker KE, Bhanot G, Gordenin DA, et al. The somatic genomic landscape of chromophobe renal cell carcinoma. Cancer Cell. 2014; 26:319-330.

3. Chen F, Zhang Y, Senbabaoglu Y, Ciriello G, Yang L, Reznik E, Shuch B, Micevic G, De Velasco G, Shinbrot E, Noble MS, Lu Y, Covington KR, et al. Multilevel Genomics-Based Taxonomy of Renal Cell Carcinoma. Cell Rep. 2016; 14:2476-2489.

4. Pelicano H, Carney D, Huang P. ROS stress in cancer cells and therapeutic implications. Drug Resist Updat. 2004; 7:97-110. 
5. Brigelius-Flohe R, Flohe L. Basic principles and emerging concepts in the redox control of transcription factors. Antioxid Redox Signal. 2011; 15:2335-2381.

6. Hayes JD, McMahon M. NRF2 and KEAP1 mutations: permanent activation of an adaptive response in cancer. Trends Biochem Sci. 2009; 34:176-188.

7. Sporn MB, Liby KT. NRF2 and cancer: the good, the bad and the importance of context. Nat Rev Cancer. 2012; 12:564-571.

8. Lee DF, Kuo HP, Liu M, Chou CK, Xia W, Du Y, Shen J, Chen CT, Huo L, Hsu MC, Li CW, Ding Q, Liao TL, et al. KEAP1 E3 ligase-mediated downregulation of NF-kappaB signaling by targeting IKKbeta. Mol Cell. 2009; 36:131-140.

9. Tian H, Zhang B, Di J, Jiang G, Chen F, Li H, Li L, Pei D, Zheng J. Keap1: one stone kills three birds Nrf2, IKKbeta and Bcl-2/Bcl-xL. Cancer Lett. 2012; 325:26-34.

10. Ooi A, Dykema K, Ansari A, Petillo D, Snider J, Kahnoski R, Anema J, Craig D, Carpten J, Teh BT, Furge KA. CUL3 and NRF2 mutations confer an NRF2 activation phenotype in a sporadic form of papillary renal cell carcinoma. Cancer Res. 2013; 73:2044-2051.

11. Srinivasan R, Ricketts CJ, Sourbier C, Linehan WM. New strategies in renal cell carcinoma: targeting the genetic and metabolic basis of disease. Clin Cancer Res. 2015; 21:10-17.

12. Muscarella LA, Parrella P, D'Alessandro V, la Torre A, Barbano R, Fontana A, Tancredi A, Guarnieri V, Balsamo T, Coco M, Copetti M, Pellegrini F, De Bonis P, et al. Frequent epigenetics inactivation of KEAP1 gene in non-small cell lung cancer. Epigenetics. 2011; 6:710-719.

13. Muscarella LA, Barbano R, D'Angelo V, Copetti M, Coco M, Balsamo T, la Torre A, Notarangelo A, Troiano M, Parisi S, Icolaro N, Catapano D, Valori VM, et al. Regulation of KEAP1 expression by promoter methylation in malignant gliomas and association with patient's outcome. Epigenetics. 2011; 6:317-325.

14. Barbano R, Muscarella LA, Pasculli B, Valori VM, Fontana A, Coco M, la Torre A, Balsamo T, Poeta ML, Marangi GF, Maiello E, Castelvetere M, Pellegrini F, et al. Aberrant Keap1 methylation in breast cancer and association with clinicopathological features. Epigenetics. 2013; 8:105-112.

15. Solis LM, Behrens C, Dong W, Suraokar M, Ozburn NC, Moran CA, Corvalan AH, Biswal S, Swisher SG, Bekele BN, Minna JD, Stewart DJ, Wistuba II. Nrf2 and Keap1 abnormalities in non-small cell lung carcinoma and association with clinicopathologic features. Clin Cancer Res. $2010 ; 16: 3743-3753$.

16. Takahashi T, Sonobe M, Menju T, Nakayama E, Mino N, Iwakiri S, Nagai S, Sato K, Miyahara R, Okubo K, Hirata T, Date $\mathrm{H}$, Wada H. Mutations in Keap1 are a potential prognostic factor in resected non-small cell lung cancer. J Surg Oncol. 2010; 101:500-506.
17. Cairns P. Renal cell carcinoma. Cancer Biomark. 2010; 9:461-473.

18. Siegel R, Naishadham D, Jemal A. Cancer statistics, 2013. CA Cancer J Clin. 2013; 63:11-30.

19. Decastro GJ, McKiernan JM. Epidemiology, clinical staging, and presentation of renal cell carcinoma. Urol Clin North Am. 2008; 35:581-592; vi.

20. Kinch L, Grishin NV, Brugarolas J. Succination of Keap1 and activation of Nrf2-dependent antioxidant pathways in FH-deficient papillary renal cell carcinoma type 2. Cancer Cell. 2011; 20:418-420.

21. Sato Y, Yoshizato T, Shiraishi Y, Maekawa S, Okuno Y, Kamura T, Shimamura T, Sato-Otsubo A, Nagae G, Suzuki H, Nagata Y, Yoshida K, Kon A, et al. Integrated molecular analysis of clear-cell renal cell carcinoma. Nat Genet. 2013; 45:860-867.

22. Guo Y, Yu S, Zhang C, Kong AN. Epigenetic regulation of Keap1-Nrf2 signaling. Free Radic Biol Med. 2015; 88: 337-349.

23. Noel S, Hamad AR, Rabb H. Reviving the promise of transcription factor Nrf2-based therapeutics for kidney diseases. Kidney Int. 2015; 88:1217-1218.

24. Wang R, An J, Ji F, Jiao H, Sun H, Zhou D. Hypermethylation of the Keap1 gene in human lung cancer cell lines and lung cancer tissues. Biochem Biophys Res Commun. 2008; 373:151-154.

25. Rimm DL. What brown cannot do for you. Nat Biotechnol. 2006; 24:914-916.

26. Larkin J, Goh XY, Vetter M, Pickering L, Swanton C. Epigenetic regulation in RCC: opportunities for therapeutic intervention? Nat Rev Urol. 2012; 9:147-155.

27. Rydzanicz M, Wrzesiński T, Bluyssen HA, Wesoły J. Genomics and epigenomics of clear cell renal cell carcinoma: recent developments and potential applications. Cancer Lett. 2013; 341:111-126.

28. Forbes SA, Beare D, Gunasekaran P, Leung K, Bindal N, Boutselakis H, Ding M, Bamford S, Cole C, Ward S, Kok CY, Jia M, De T, et al. COSMIC: exploring the world's knowledge of somatic mutations in human cancer. Nucleic Acids Res. 2015; 43:D805-811.

29. Menegon S, Columbano A, Giordano S. The Dual Roles of NRF2 in Cancer. Trends Mol Med. 2016; 22:578-593.

30. Lu MC, Ji JA, Jiang ZY, You QD. The Keap1-Nrf2-ARE Pathway As a Potential Preventive and Therapeutic Target: An Update. Med Res Rev. 2016; 36:924-963.

31. Parrella P, Poeta ML, Gallo AP, Prencipe M, Scintu M, Apicella A, Rossiello R, Liguoro G, Seripa D, Gravina C, Rabitti C, Rinaldi M, Nicol T, et al. Nonrandom distribution of aberrant promoter methylation of cancer-related genes in sporadic breast tumors. Clin Cancer Res. 2004; 10:5349-5354. 